\title{
Knot and Tonk \\ Nasty connectives on many-valued truth-tables for classical sentential logic
}

\author{
Tim Button \\ button@cantab.net \\ University of Cambridge
}

November 28, 2015

This paper is due to be published in Analysis. This is a pre-print and may be subject to minor alterations. The authoritative version should be sought from Analysis.

\section{Preliminaries}

Some connectives cause havoc when added to a language. One such connective is Prior's (1960) Tonk, defined via these inference rules:

$$
\frac{\varphi}{\varphi \mathrm{t} \psi} \mathrm{tr}^{\mathrm{\varphi t} \psi} \mathrm{te}_{\mathrm{tE}}
$$

If you add Tonk to a language, then triviality ensues. Of course, languages containing Tonk are perfectly good objects of formal study. However, languages are not just objects of study, but things we use, and we must refuse to add Tonk to the languages we use. So this raises a general question: Which connectives should we be willing to add to the languages we use?

One kind of answer has the following shape: We should we willing to add exactly those connectives with suitably assigned meanings. Such an answer demands a general discussion of how connectives are assigned meanings, and here there are two very broad camps. Inferentialists focus on the inference rules governing connectives, whereas semanticists focus on their semantic conditions.

The case of Tonk places inferentialists on the back foot. Tonk cannot be given semantic conditions, so semanticists have no reason even to entertain adding Tonk to their languages; indeed, they may go so far as to deny that Tonk is even meaningful. By contrast, Tonk has perfectly well-defined inference rules. So, at the very least, inferentialists need to tell us which kinds of inference rules succeed in 'suitably' assigning meanings.

Inferentialists have said many things in response. However, my aim in this paper is not to consider those responses, but rather to show that semanticists are in a very similar position to inferentialists. Crudely: semanticists face their 'very own Tonk'. Slightly 
more precisely: there are connectives which implement perfectly well-defined semantic functions, but which we should refuse to add to the languages we use. Such nasty connectives somewhat even the score between inferentialists and semanticists.

In the next section, I use many-valued truth-tables for classical sentential logic to define a nasty connective, Knot. In $\S_{3}$, I argue that we should refuse to add Knot to the languages we use. In $\$ 4$, I show that Knot reverses the standard dialectic surrounding Tonk, and also yields a novel solution to the problem of many-valued truth-tables for classical sentential logic. I reserve all proofs for $\$ 5$.

\section{Many-valued truth-tables and Knot}

We are all familiar with the usual system of truth-tables for classical sentential logic:

$$
\begin{array}{l|ll|lll|lll|ll} 
& \neg & \wedge & 1 & 0 & \vee & 1 & 0 & \rightarrow & 1 & 0 \\
\hline 1 & 0 & 1 & 1 & 0 & 1 & 1 & 1 & 1 & 1 & 0 \\
\circ & 1 & \circ & 0 & 0 & 0 & 1 & 0 & 0 & 1 & 1
\end{array}
$$

But here is a less usual system of truth-tables, due to Church (1944: 494, 1953: 50; see also Carnap 1943: 73ff.; Smiley 1996: 6; Koslow 2010: 125-6):

\begin{tabular}{l|l} 
& $\neg$ \\
\hline $\mathbf{1}$ & $\circ$ \\
$a$ & $b$ \\
$b$ & $a$ \\
$\circ$ & $\mathbf{1}$
\end{tabular}

\begin{tabular}{l|llll}
$\wedge$ & $\mathbf{1}$ & $a$ & $b$ & 0 \\
\hline $\mathbf{1}$ & $\mathbf{1}$ & $a$ & $b$ & 0 \\
$a$ & $a$ & $a$ & 0 & 0 \\
$b$ & $b$ & 0 & $b$ & 0 \\
$\circ$ & 0 & 0 & 0 & 0
\end{tabular}

\begin{tabular}{l|llll}
$\vee$ & 1 & $a$ & $b$ & 0 \\
\hline $\mathbf{1}$ & $\mathbf{1}$ & $\mathbf{1}$ & $\mathbf{1}$ & $\mathbf{1}$ \\
$a$ & $\mathbf{1}$ & $a$ & $\mathbf{1}$ & $a$ \\
$b$ & $\mathbf{1}$ & $\mathbf{1}$ & $b$ & $b$ \\
$\circ$ & $\mathbf{1}$ & $a$ & $b$ & $\circ$
\end{tabular}

\begin{tabular}{l|llll}
$\rightarrow$ & 1 & $a$ & $b$ & 0 \\
\hline $\mathbf{1}$ & $\mathbf{1}$ & $a$ & $b$ & 0 \\
$a$ & 1 & 1 & $b$ & $b$ \\
$b$ & 1 & $a$ & 1 & $a$ \\
$\circ$ & 1 & 1 & 1 & 1
\end{tabular}

Suppose we treat 1 as the only designated semantic value (hence the bold font). Intuitively, this is to say that some premises entail a conclusion iff, whenever all the premises have value 1 , the conclusion also has value 1 . We have now defined a logic using our fourvalued truth-tables. (Throughout, I take a logic just to be any two-place relation which relates sets of sentences - think 'premises'-to individual sentences - think 'conclusion.) This four-valued definition may be unfamiliar, but the logic it defines is very familiar indeed: it is our old friend, classical sentential logic. Otherwise put: the two different systems of truth-tables yield the very same logic (see Theorem 1).

The four-valued system, though, provides us with the opportunity to introduce some genuinely new connectives. So, let Knot, symbolised with 8, be the one-place connective which simply swaps $a$ with $b$, but leaves 1 and o unchanged. ${ }^{1}$ This truth-table illustrates the behaviour of Knot, and of a few related formulas.

\footnotetext{
${ }^{1}$ An anonymous referee noted that Knot can be regarded as a Conflation operator, in Fitting's sense (1994: 123). However, that comparison is imperfect, since this four-valued system is distinctively Boolean (see $\$_{5}$ ), whereas Fitting defines Conflation for systems which cannot have Boolean negation.
} 


\begin{tabular}{c|ccccccc}
$\varphi$ & $8 \varphi$ & $\neg \varphi$ & $\varphi \rightarrow \varphi$ & $\varphi \vee \neg \varphi$ & $\varphi \rightarrow R \varphi$ & $\neg(\varphi \rightarrow \ell \varphi)$ & $\neg \neg(\varphi \rightarrow R \varphi)$ \\
\hline $\mathbf{1}$ & $\mathbf{1}$ & 0 & $\mathbf{1}$ & $\mathbf{1}$ & $\mathbf{1}$ & 0 & $\mathbf{1}$ \\
$a$ & $b$ & $b$ & $\mathbf{1}$ & $\mathbf{1}$ & $b$ & $a$ & $b$ \\
$b$ & $a$ & $a$ & $\mathbf{1}$ & $\mathbf{1}$ & $a$ & $b$ & $a$ \\
$\circ$ & 0 & $\mathbf{1}$ & $\mathbf{1}$ & $\mathbf{1}$ & $\mathbf{1}$ & 0 & $\mathbf{1}$
\end{tabular}

Now let $\frac{\Sigma}{\bar{q}}$ be the logic defined using our four-valued truth-tables, for sentences whose only connectives are among $\neg, \wedge, \vee, \rightarrow$ and $\ell$. Thanks to the presence of Knot, the logic $\Sigma_{8}$ violates four common logical principles (these principles are defined in $\$_{5}$ ).

First, $\models_{8}$ violates the Substitutivity of Equivalents; that is it violates the principle that substituting a subsentence for a logically equivalent subsentence never affects entailment. Glancing at our table, we see that $p$ and $\Omega p$ are logically equivalent in the sense

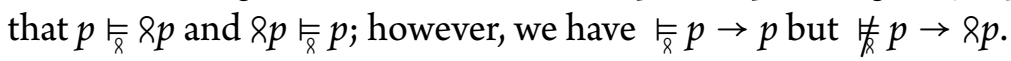

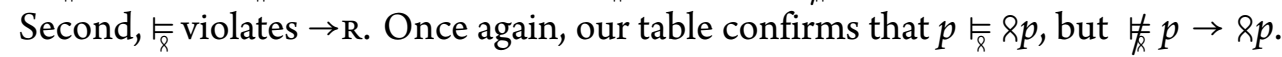
We can equally regard this as a failure of $\rightarrow$-introduction.

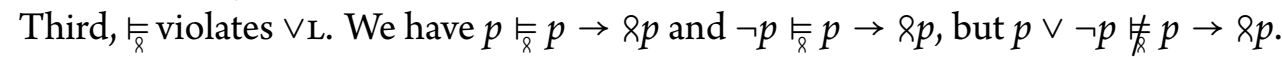
We can equally regard this as a failure of $\vee$-elimination.

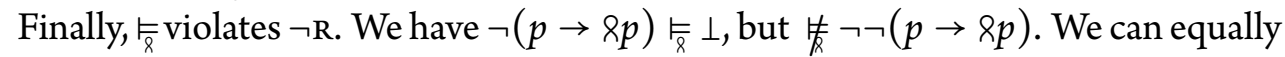
regard this as a failure of $\neg$-introduction.

\section{Knot is nasty}

I have just defined a new connective, Knot. Given the discussion of $\S_{1}$, I should now consider whether we should be willing to add Knot to the languages we use. I think that we should refuse to add Knot to our language, since doing so would force us to adopt an undesirable logic.

To be clear, Knot is less horrible than Tonk. Adding Tonk to a language leads to logical triviality, and $\Sigma_{\bar{\gamma}}$ is certainly not trivial. However, the lesson of $\S_{2}$ is that, if we add Knot to our language, then we must abandon the Substitutivity of Equivalents, $\rightarrow R, V_{L}$ and $\neg \mathrm{R}$. Classical logic validates all four principles. By contrast, logics lacking all four principles are extremely weak; too weak, I think, for us to want to use them.

Admittedly, such weak logics are not unheralded. Indeed,,$\frac{5}{8}$ has an exactly similar modal counterpart. ${ }^{2}$ Let $\mathcal{T}$ be a Kripke frame containing two worlds, $w_{1}$ and $w_{2}$, both of which can see the other, and neither of which can see itself. A valuation on $\mathcal{T}$ is then an assignment of each atomic sentence to one of four possible values (intuitively, the worlds 'where the atomic sentence is true'), namely: $1=\left\{w_{1}, w_{2}\right\}, a=\left\{w_{1}\right\}, b=\left\{w_{2}\right\}$

(In detail: Fitting requires a partial order on the semantic values, with a top and bottom element, such that $x \leq y \Rightarrow \neg x \leq \neg y$; but if $t$ is the top element under $\leq$, then $\neg t \leq t$ and hence $t=\neg \neg t \nless \neg t$.)

${ }^{2}$ Many thanks to an anonymous referee for supplying this construction. 
and $o=\varnothing$. Now let $\vDash$ be the global consequence relation for $\mathcal{T}$, i.e.: $\Sigma$ 辰 $\rho$ iff no valuation on $\mathcal{T}$ makes every $\sigma \in \Sigma$ true at all worlds whilst making $\rho$ false at some world.

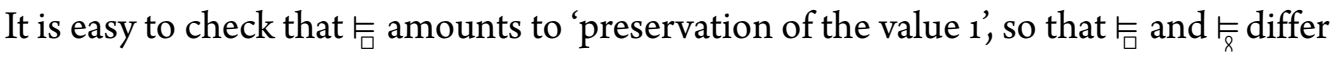
only in that one uses the sign ' $\square$ ' where the other uses the sign ' 8 '.

I mention this construction, since some supervaluationists about vagueness hold that the correct logic for vagueness is obtained by adopting a suitable global consequence relation and reading ' $\square$ ' as a determinacy operator (see Williamson 1994: ch.5). Given the equivalence between the two logics $\frac{5}{\square}$ and $\sqrt{8}$, then, it is worth noting that Knot cannot plausible be read as a determinacy operator. Since $\Sigma_{\bar{\gamma}} \ell \varphi \vee \Omega \neg \varphi$, reading Knot as a determinacy operator would license the general principle: either $x$ is definitely pink, or definitely $x$ is not pink. Similarly, since $\frac{\bar{\gamma}}{\phi} \leftrightarrow 88 \varphi$, it would license the principle: $x$ is pink iff $x$ is definitely definitely pink. Both principles violate the vagueness of pink.

This rules out one potential reason for speaking a language which includes Knot. It falls short of showing that no one could ever find a reason to speak a language including Knot. Nonetheless, I suspect that many philosophers will agree that we should no more add Knot than add Tonk to our language, since doing so would leave us with an undesirable logic. In any case, I assume as much for the remainder of the paper.

\section{$4 \quad$ Knot lends support to inferentialism}

Having refused to add Knot to our language, we may want to say more to justify our decision. In $\$_{1}$, I noted that certain philosophers are prepared to add exactly those connectives with 'suitably' assigned meanings. I then distinguished two broad approaches for considering the meanings of connectives: inferentialists focus on inference rules, whilst semanticists focus on semantic conditions. My aim now is to show why Knot helps inferentialists whilst raising problems for semanticists.

Knot clearly implements a well-defined semantic function. So semanticists need, at least, to tell us which kinds of semantic constraints succeed in 'suitably' assigning meanings. The situation here reverses the dialectic concerning Tonk perfectly: since Tonk has well-defined inference rules, inferentialists need, at least, to tell us which kinds of inference rules succeed in 'suitably' assigning meanings.

This reversal of fortunes continues. Just as semanticists are untroubled by Tonk, so inferentialists are untroubled by Knot. To see why, note that if Knot were somehow added to our language, then it would have to cause the loss of other inference rules. (For example: the inferentialist presumably insists that the conditional is governed by the rules of $\rightarrow$-elimination and $\rightarrow$-introduction, but we saw in $\$_{2}$ that $\rightarrow$-introduction fails in the presence of Knot.) However, the inferentialist has stipulated that these inference rules are to hold always and without exception. The inferentialist can therefore legitimately maintain that Knot simply cannot be added to our language, given the (in- 
ferentially specified) meanings of the other connectives.

This raises a further parallel between inferentialist reactions to Knot and semanticist reactions to Tonk. Semanticists sometimes allege that the natural deduction rules for Tonk fail even to define a meaningful connective, on the grounds that Tonk cannot be given semantic conditions. By exactly the same token, inferentialists might allege that the semantic conditions for Knot fail even to define a meaningful connective, on the grounds that Knot cannot be given natural-deduction-style inference rules, given the (inferentially specified) meanings of the other connectives.

In any case, inferentialists have highly principled reasons for refusing to incorporate Knot into their language. And it gets even better for inferentialists. Not only does Knot cause them no problems; their principled story concerning Knot suggests a new line of response to what we might call the problem of many-valued truth-tables. ${ }^{3}$

That problem arises as follows. As we saw in $\$ 2$, there are systems of truth-tables with more than two truth-values, which nonetheless characterise classical sentential logic. Call these many-valued truth-tables for classical sentential logic. Consequently, the classical inference rules for the connectives $\neg, \wedge, \vee$ and $\rightarrow$ fail to pin down the twovalued truth-tables uniquely (up to isomorphism). From this, one might conclude that the connectives' inference rules fail to determine their meanings. I say 'might', because it is not entirely obvious that the availability of many-valued truth-tables amounts to a problematic indeterminacy of meaning. But if it does, then inferentialism fails.

However, if inferentialists want to pin down the truth-tables for classical sentential logic, they can. Suppose they stipulate: It is impossible to add further connectives to our language which would cause violations of any of $\rightarrow R, \vee L$ or $\neg R$. This stipulation is perfectly in the spirit of inferentialism: it invokes no semantic notions, and it simply repeats the inferentialist's principled reasons for denying that we should (or could) add Knot to our language. However, when assessed at the semantic level, it guarantees that any system of truth-tables has the usual relation between designated and undesignated values, i.e. (see Theorem 3):

$$
\begin{aligned}
& \neg \varphi \text { is designated iff } \varphi \text { is undesignated } \\
& \varphi \wedge \psi \text { is designated iff both } \varphi \text { and } \psi \text { are designated } \\
& \varphi \vee \psi \text { is designated iff either } \varphi \text { or } \psi \text { is designated } \\
& \neg \varphi \vee \psi \text { is designated iff either } \varphi \text { is undesignated or } \psi \text { is designated }
\end{aligned}
$$

Furthermore, consider the stipulation: It is impossible to add further connectives to our language which would cause violations of the Substitutivity of Equivalents. This stipulation again seems in the spirit of inferentialism: it mentions only inferential concerns, and inferentialists can insist upon Substitutivity as a constraint on inference (perhaps as a

\footnotetext{
${ }^{3}$ This relates to Carnap's categoricity problem (see Carnap 1943: 73ff.; Smiley 1996: 6; Koslow 2010: 125-6).
} 
structural rule). However, assessed at the semantic level, this rule uniquely determines the usual, two-valued truth-tables (up to isomorphism; see Theorem 6).

To summarise: a single inferentialist idea explains both why we should refuse to add Knot to our language, and dissolves the problem of many-valued truth-tables. Semanticists, however, still owe us a discussion of Knot.

Of course, just as inferentialists have said things about Tonk, so semanticists will have things to say about Knot. It will be interesting to hear what they have to say. In the meantime, Knot somewhat evens the score between inferentialists and semanticists.

\section{Technicalities}

To close this paper, I shall explore the technicalities surrounding nasty connectives and many-valued truth-tables for classical sentential logic.

I shall consider multiple languages for sentential logics. The atomic sentences of these languages are always just $p$ and $q$, with arbitrary primes. The set of connectives for these languages always includes $\{\neg, \wedge, \vee, \rightarrow\}$. The sentence-formation rules are standard, so that a language's set of connectives determines its sentences. I use lower-case Greek letters for arbitrary sentences, upper-case Greek letters for sets of sentences, and treat ' $\perp$ ' as an abbrevation for ' $(p \wedge \neg p)$ '.

Let $L$ be any set of connectives. An $L$-semantic-pair is any pair $\langle\mathcal{A}, D\rangle$ such that $\mathcal{A}$ is an algebraic L-structure-i.e. each $n$-place connective in $\mathrm{L}$ is interpreted as an $n$-place function over $\mathcal{A}$ 's domain, $A$ - and $D \subseteq A$. The intuitive idea is to define a logic for the sentences whose connectives are members of $\mathrm{L}$, by generating truth-tables from $\mathcal{A}$ 's functions and treating $D$ as the set of designated values.

In detail, an interpretation on an $L$-structure $\mathcal{A}$ is any map, $v$, from the set of atomic sentences to $A$, which assigns values to complex sentences via these clauses:

$$
\begin{array}{rlrl}
v(\triangle \varphi) & =\Delta v(\varphi) & & \text { for each 1-place connective } \triangle \in \mathrm{L} \\
v(\phi \triangleleft \psi) & =v(\varphi) \triangleleft v(\psi) & & \text { for each 2-place connective } \triangleleft \in \mathrm{L} \\
\text { etc. } & &
\end{array}
$$

In each clause, the same symbol occurs on the left, to indicate a connective of the object language, and on the right, to indicate a function on $\mathcal{A}$. These clauses guarantee that our object-language connectives are truth-functional, and so are associated with characteristic truth-tables. To obtain our logic, we now define: $\Sigma \frac{\mathscr{R}}{\bar{D}} \rho$ iff there is no interpretation $v$ on $\mathcal{A}$ such that $v(\sigma) \in D$ for all $\sigma \in \Sigma$ but $v(\rho) \notin D$.

It might help to connect this framework to the four-valued truth-tables of $\$_{2}$. So, consider the Boolean algebra whose Hasse diagram is: 


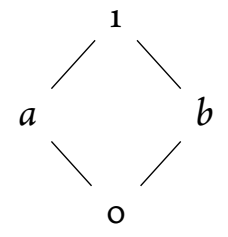

Expand this to a $\{\neg, \wedge, \vee, \rightarrow\}$-structure by defining $(x \rightarrow y)={ }_{\mathrm{df}}(\neg x \vee y)$. By considering the interpretations on this structure, we obtain the initial four-valued truth-tables from $\$_{2}$. Expand this even further, to an $\{\neg, \wedge, \vee, \rightarrow, \ell\}$-structure, by defining $\ell_{1}=1$, $8 a=b, 8 b=a$ and $80=0$; we now obtain the truth-table for Knot. Finally, if we call

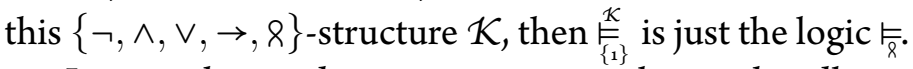

It is worth introducing some terminology to handle such uses of Boolean algebras. For any signature $\mathrm{L} \supseteq\{\neg, \wedge, \vee, \rightarrow\}$, say that an L-structure $\mathcal{A}$ is canonically-Boolean iff both $(x \rightarrow y)=(\neg x \vee y)$ in $\mathcal{A}$ and $\mathcal{A}$ 's $\{\neg, \wedge, \vee\}$-reduct is a Boolean algebra. ${ }^{4}$ In these terms, the $\{\neg, \wedge, \vee, \rightarrow, 8\}$-structure $\mathcal{K}$ is canonically-Boolean.

Most semantic-pairs are not very interesting, but some will be extremely important to us. Using $\vDash$ for our familiar classical sentential logic, say that a semantic-pair $\langle\mathcal{A}, D\rangle$ is proto-classical just in case the relations $\vDash$ and $\frac{\mathscr{A}}{\bar{D}}$ are coextensive over any sentences whose only connectives are among $\neg, \wedge, \vee$ and $\rightarrow$. Intuitively, the logic of a proto-classical semantic-pair behaves exactly like classical sentential logic, provided we restrict our attention to sentences containing the usual connectives. My interest lies in what happens when we consider other connectives.

To explore this, it will help to consider some general principles that might govern a logic. I call the following fourteen principles the classical principles:

$$
\begin{aligned}
& \bar{\varphi}^{\text {REP }} \quad \frac{\Sigma \vdash \varphi \quad \varphi, \Delta \vdash \rho}{\Sigma, \Delta \vdash \rho} \text { CUT } \\
& \frac{\Sigma \vdash \rho}{\varphi, \Sigma \vdash \rho} \text { Lw } \quad \frac{\Sigma \vdash \perp}{\Sigma \vdash \rho} \mathrm{RW} \\
& \frac{\varphi, \psi, \Sigma \vdash \rho}{\varphi \wedge \psi, \Sigma \vdash \rho} \wedge \mathrm{L} \quad \frac{\Sigma \vdash \varphi \quad \Sigma \vdash \psi}{\Sigma \vdash \varphi \wedge \psi} \wedge \mathrm{R} \\
& \frac{\varphi, \Sigma \vdash \rho \quad \psi, \Sigma \vdash \rho}{\varphi \vee \psi, \Sigma \vdash \rho} \vee \mathrm{L} \quad \frac{\Sigma \vdash \varphi}{\Sigma \vdash \varphi \vee \psi} \vee_{\mathbf{R}_{1}} \quad \frac{\Sigma \vdash \varphi}{\Sigma \vdash \psi \vee \varphi} \vee_{\mathbf{R}_{2}}
\end{aligned}
$$

\footnotetext{
${ }^{4} \mathrm{Cf}$. Church (1953: $\mathrm{n} \cdot 3$ ). When we have two signatures $\mathrm{L}^{+} \supseteq \mathrm{L}$ and an $\mathrm{L}^{+}$-structure $\mathcal{M}^{+}$, we can obtain a unique $\mathrm{L}$-structure $\mathcal{M}$ by ignoring the interpretations of all the symbols in $\mathrm{L}^{+} \backslash \mathrm{L}$; we then say that $\mathcal{M}$ is $\mathcal{M}^{+}$'s $\mathrm{L}$-reduct, and that $\mathcal{M}^{+}$is a signature-expansion of $\mathcal{M}$.
} 


$$
\begin{array}{lcl}
\frac{\Sigma \vdash \varphi}{\neg \varphi, \Sigma \vdash \perp} \neg \mathrm{L} & \frac{\varphi, \Sigma \vdash \perp}{\Sigma \vdash \neg \varphi} \neg \mathrm{R} & \\
\frac{\varphi, \Sigma \vdash \psi}{\Sigma \vdash \varphi \rightarrow \psi} \rightarrow \mathrm{R} & \frac{\Sigma \vdash \varphi \quad \psi, \Sigma \vdash \rho}{\varphi \rightarrow \psi, \Sigma \vdash \rho} \rightarrow \mathrm{L} &
\end{array}
$$

Classical sentential logic validates $\wedge \mathrm{R}$, for example, in that if both $\Sigma \vDash \varphi$ and $\Sigma \vDash \psi$, then $\Sigma \vDash \varphi \wedge \psi$. Indeed, the classical principles are so named, because they provide a sound and complete sequent-calculus-style proof theory for classical sentential logic.

In addition to the classical principles, I should also mention the Substitutivity of Equivalents, more briefly called suB:

$$
\begin{array}{ccl}
\Sigma \vdash \rho \quad \varphi \vdash \psi \quad \psi \vdash \varphi & \text { suB } \quad \text { when } \rho \text { and } \rho^{*} \text { differ only in that one contains an instance } \\
& \sum \vdash \rho^{*} & \text { of } \varphi \text { as a subsentence where the other contains } \psi
\end{array}
$$

Classical sentential logic validates all of the classical principles and sub. My aim is to classify the circumstances under which proto-classical semantic-pairs can violate either a classical principle or suB.

I shall start by generalising the construction of many-valued truth-tables from $\$ 2 .{ }^{5}$

Theorem 1: Let $\mathcal{A}$ be canonically-Boolean with $D \subseteq A$. Then $\langle\mathcal{A}, D\rangle$ is proto-classical iff $D$ is a filter on $A$.

Proof. Left-to-right. Fix $\mathcal{A}$ as described, and suppose $D \subseteq A$ is not a filter. There are three possible cases to consider, and in each case $\langle\mathcal{A}, D\rangle$ is not proto-classical:

Case 1. $\circ \in D$. Then $\frac{\mathscr{R}}{\bar{D}} p \wedge \neg p$, since $v(p \wedge \neg p)=o \in D$ for all $v$.

Case 2. $a \in D$ and $a \leq b$ but $b \notin D$, for some $a, b \in A$. Then $p$ 哗 $p \vee q$, since $v(p)=a$ and $v(q)=b$ gives $v(p \vee q)=b \notin D$.

Case 3. $a, b \in D$ but $a \wedge b \notin D$, for some $a, b \in A$. Then $p, q$ 男 $p \wedge q$, since $v(p)=a$ and $v(q)=b$ gives $v(p \wedge q)=a \wedge b \notin D$.

Right-to-left. Fix $\mathcal{A}$ as described, with a filter $D \subseteq A$, and a set of sentences $\Sigma \cup\{\rho\}$ whose only connectives are among $\neg, \wedge, \vee$, and $\rightarrow$. We must show that $\Sigma \frac{\mathscr{A}}{\bar{D}} \rho$ iff $\Sigma \vDash \rho$.

Necessity. If $\Sigma \frac{\mathscr{A}}{\bar{D}} \rho$, then there is no interpretation $v$ on $\mathcal{A}$ such that $v(\sigma)=1$ for all $\sigma \in \Sigma$ and $v(\rho)=0$. A fortiori, there is no interpretation $v$ with range $\{0,1\}$ such that $v(\sigma)=1$ for all $\sigma \in \Sigma$ and $v(\rho)=$ o. Hence $\Sigma \vDash \rho$

Sufficiency. If $\Sigma \vDash \rho$, then there is a sequent-style proof, using only the classical principles, whole last line is $\Sigma_{\mathrm{o}} \vdash \rho$, for some finite $\Sigma_{\mathrm{o}} \subseteq \Sigma$. I claim that, for any sequent

${ }^{5}$ This extends Church's (1944: 494, 1953: 41-2) observation that any Boolean algebra yields a truth-table for classical sentential logic if we treat either $\{1\}$ or some ultrafilter on the algebra as designated. (Church describes the truth-tables that result from ultrafilters as 'normal in the sense of Carnap'.) Recall that $D$ is a filter provided (1) o $\notin D$; (2) if $a \in D$ and $a \leq b$ then $b \in D$; and (3) if $a, b \in D$ then $a \wedge b \in D$. $D$ is an ultrafilter provided also (4) either $a \in D$ or $\neg a \in D$ for all algebraic elements $a$. 
$\Phi \vdash \tau$ in this proof, we have $v(\wedge \Phi) \leq v(\tau)$ for any interpretation $v$ on $\mathcal{A}$. The claim is established by induction on height. For example, consider a use of $\vee_{L}$ :

$$
\frac{\varphi, \Gamma \vdash \tau \quad \psi, \Gamma \vdash \tau}{\varphi \vee \psi, \Gamma \vdash \tau} \vee \mathrm{L}
$$

Suppose, for induction, that $v(\varphi \wedge \wedge \Gamma) \leq v(\tau)$ and $v(\psi \wedge \wedge \Gamma) \leq v(\tau)$. Then by invoking the recursion clauses for $v$ and the distributivity laws of Boolean algebras:

$$
v((\varphi \vee \psi) \wedge \bigwedge \Gamma))=v(\varphi \wedge \bigwedge \Gamma) \vee v(\psi \wedge \bigwedge \Gamma) \leq v(\tau) \vee v(\tau)=v(\tau)
$$

as required. The other cases are similar, establishing that $v(\wedge \Phi) \leq v(\tau)$. Since $D$ is closed upwards and closed under finite meet, $\Phi \frac{\mathscr{R}}{\bar{D}} \tau$. Hence $\Sigma_{\mathrm{o}} \frac{\mathscr{P}}{\bar{D}} \rho$ and so $\Sigma \frac{\mathcal{A}}{\bar{D}} \rho$.

However, Theorem 1 does not exhaust the proto-classical semantic-pairs. Let $\mathcal{F}$ be an $\{\neg, \wedge, \vee, \rightarrow\}$-structure with domain $\{1, a, o\}$ and functions given by

\begin{tabular}{l|ll|lllll|lllll|lll} 
& $\neg$ & $\wedge$ & 1 & $a$ & 0 & $\vee$ & 1 & $a$ & 0 & $\rightarrow$ & 1 & $a$ & 0 \\
\hline 1 & 0 & 1 & 1 & 0 & 0 & 1 & 1 & 1 & 1 & 1 & 1 & 0 & 0 \\
$a$ & 1 & $a$ & 0 & 0 & 0 & $a$ & 1 & 0 & 0 & $a$ & 1 & 1 & 1 \\
$\circ$ & 1 & 0 & 0 & 0 & 0 & & 0 & 1 & 0 & 0 & 0 & 1 & 1 & 1
\end{tabular}

Intuitively, $a$ is a frivolous alternative to o in the two-valued truth-tables, hence $\langle F,\{1\}\rangle$ is proto-classical. But $\mathcal{F}$ is not canonically-Boolean, since $\neg \neg a=0 \neq a$.

We now have a sense of the range of proto-classical semantic-pairs. The next task is to generalise the construction of $\operatorname{Knot}$ from $\S_{2}$. Say that a semantic-pair $\langle\mathcal{A}, D\rangle$ is knotty iff there is some signature-expansion $\mathcal{B}$ of $\mathcal{A}$ such that $\frac{\mathcal{B}}{\bar{D}}$ violates all of SUB, $\rightarrow \mathrm{R}, \mathrm{VL}$ and $\neg$ R. Intuitively, a semantic-pair is knotty just when we can add a single new connective which would be exactly as disruptive as Knot. The next Theorem gives us a sufficient condition for knottiness:

Theorem 2: If $\langle\mathcal{A}, D\rangle$ is proto-classical and there is some $a \in A$ such that $a, \neg a \notin D$, then $\langle\mathcal{A}, D\rangle$ is knotty.

Proof. Let + be a one-place connective not in $\mathcal{A}$ 's signature. Expand $\mathcal{A}$ to $\mathcal{B}$ by defining + on $A$ as follows: $+a=\neg a,+\neg a=a$ and $+x=x$ otherwise. Here is a compressed truthtable for some pertinent formulas for the logic $\frac{B}{\frac{B}{D}}$ :

\begin{tabular}{c|ccccc}
$\varphi$ & $\varphi \rightarrow \varphi$ & $\varphi \vee \neg \varphi$ & $\varphi \rightarrow+\varphi$ & $\neg(\varphi \rightarrow+\varphi)$ & $\neg \neg(\varphi \rightarrow+\varphi)$ \\
\hline$a$ or $\neg a$ & $\checkmark$ & $\checkmark$ & $\times$ & $\times$ & $\times$ \\
otherwise & $\checkmark$ & $\checkmark$ & $\checkmark$ & $\times$ & $\checkmark$
\end{tabular}


A tick on a row indicates that the sentence is designated (i.e. $v$ assigns it a member of $D)$ for the stated value of $\varphi$; a cross on a row indicates that it is undesignated for that value. For example, the table indicates: if $v(\varphi) \in\{a, \neg a\}$, then $v(\varphi \rightarrow+\varphi) \notin D$. To see this, observe that $\varphi \rightarrow \neg \varphi \frac{\mathcal{B}}{\bar{D}} \neg \varphi$, since $\langle\mathcal{B}, D\rangle$ is proto-classical; so if $v(\varphi) \in\{a, \neg a\}$ then $v(\neg \varphi)=\neg v(\varphi) \notin D$, so that $v(\varphi \rightarrow+\varphi)=v(\varphi \rightarrow \neg \varphi) \notin D$. Equally, the table indicates: if $v(\varphi) \notin\{a, \neg a\}$, then $v(\varphi \rightarrow+\varphi) \in D$. To see this, observe that $\frac{\mathcal{B}}{\bar{D}} \varphi \rightarrow \varphi$, since $\langle\mathcal{B}, D\rangle$ is proto-classical; and so if $v(\varphi) \notin\{a, \neg a\}$ then $v(+\varphi)=+v(\varphi)=v(\varphi)$, so that $v(\varphi \rightarrow+\varphi)=v(\varphi \rightarrow \varphi) \in D$. The other entries on the table can be confirmed similarly. It follows that:

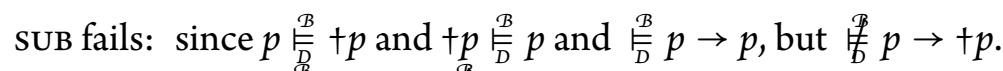

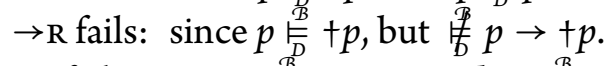

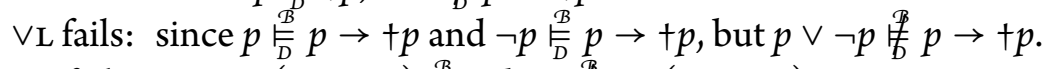

$\neg \mathrm{R}$ fails: since $\neg(p \rightarrow+p) \underset{\bar{D}}{\frac{B}{D}} \perp$, but $\frac{g^{\prime}}{D} \neg \neg(p \rightarrow+p)$.

Note that Theorem 2 assumes only that $\langle\mathcal{A}, D\rangle$ is proto-classical, and not that $\mathcal{A}$ is

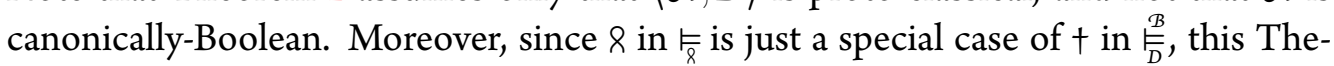
orem properly generalises the construction of Knot.

The key to Theorem 2 is the existence of an undesignated element whose negation is also undesignated. Banning this case pins down the behaviour of the logic significantly, as the next three results show.

Theorem 3: Let $\langle\mathcal{A}, D\rangle$ be proto-classical, with no $a \in A$ such that $a, \neg a \notin D$. Then: $\neg a \in D$ iff $a \notin D ; a \wedge b \in D$ iff both $a \in D$ and $b \in D ; a \vee b \in D$ iff either $a \in D$ or $b \in D$; and $a \rightarrow b \in D$ iff either $a \notin D$ or $b \in D$.

Proof. Negation. Since $\langle\mathcal{A}, D\rangle$ is proto-classical, $A$ has at least one undesignated value, say $c$. If there were some $a \in D$ such that both $a, \neg a \in D$, then setting $v(p)=a$ and $v(q)=c$ we would have $p, \neg p$ 圊 $q$, contradicting proto-classicality.

Conjunction. This holds since $p, q \frac{\mathscr{R}}{\bar{D}} p \wedge q$ and $p \wedge q \frac{\mathscr{R}}{\bar{D}} p$ and $p \wedge q \frac{\mathscr{A}}{\bar{D}} q$.

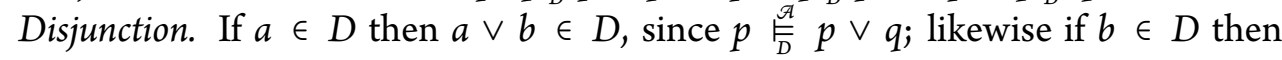
$a \vee b \in D$. Conversely, if $a, b \notin D$, then $\neg a, \neg b \in D$ by the clause for Negation; and since $\neg p, p \vee q \frac{\mathcal{H}}{\bar{D}} q$, we must have $a \vee b \notin D$.

Conditional. Since $\langle\mathcal{A}, D\rangle$ is proto-classical, $a \rightarrow b \in D$ iff $\neg a \vee b \in D$; so this follows from the clauses of Negation and Disjunction.

With Theorem 2, this vindicates my remark that we can pin down the relations between designated and undesignated values via the stipulation: It is impossible to add further connectives to our language which would cause violations of any of $\rightarrow R, \vee_{L}$ or $\neg R$ (see $\$_{5}$ ).

The next Theorem shows that Knot-like connectives are the nastiest possible connectives which can be defined on semantic-pairs, and that our sufficient condition for knottiness (from Theorem 2) is also necessary: 
Theorem 4: Let $\langle\mathcal{A}, D\rangle$ be proto-classical. Then $\frac{\mathcal{A}}{\bar{D}}$ validates all the classical principles, except perhaps $\rightarrow R, \vee L, \neg R$. Furthermore, if there is no $a \in A$ such that $a, \neg a \notin D$, then $\frac{\mathcal{A}}{\bar{D}}$ validates all the classical principles.

Proof. I shall prove the hardest case, leaving the remainder for the reader. The case is that $\vee_{L}$ holds when there is no $a \in A$ such that $a, \neg a \notin D$. So suppose that $\varphi, \Sigma \frac{\mathscr{A}}{\bar{D}} \rho$ and $\psi, \Sigma \stackrel{\mathscr{R}}{\overline{\bar{D}}} \rho$. Suppose that $v(\sigma) \in D$ for all $\sigma \in \Sigma$, and $v(\varphi \vee \psi)=v(\varphi) \vee v(\psi) \in D$. Then by Theorem 3 either $v(\varphi) \in D$ or $v(\psi) \in D$; and either way, $v(\rho) \in D$, because both $\varphi, \Sigma \stackrel{\mathscr{P}}{\bar{D}} \rho$ and $\psi, \Sigma \stackrel{\mathscr{R}}{\bar{D}} \rho$. Hence $\varphi \vee \psi, \Sigma \stackrel{\mathscr{R}}{\bar{D}} \rho$.

Between them, then, Theorems 2 and 4 yield a necessary and sufficient condition for knottiness. Moreover, Theorems 1-4 immediately yield a nice Corollary:

Corollary 5: Let $\mathcal{A}$ be canonically-Boolean with $D \subseteq A$. Then $\langle\mathcal{A}, D\rangle$ is proto-classical but knotty iff $D$ is a filter but not an ultra-filter on $\mathcal{A}$.

Finally, even when a semantic-pair is not knotty, we can almost always find some nasty connectives; we just need to focus on failures of sub.

Theorem 6: If $\langle\mathcal{A}, D\rangle$ is proto-classical, then the following are equivalent:

(a) $\mathcal{A}$ 's $\{\neg, \wedge, \vee\}$-reduct is isomorphic to the two-element Boolean algebra

(b) for any signature-expansion $\mathcal{B}$ of $\mathcal{A}, \frac{\mathcal{B}}{\bar{D}}$ validates SUB

Proof. $(a) \Rightarrow(b)$. Just observe that $\{\neg, \wedge, \vee\}$ is expressively adequate for the twoelement Boolean algebra.

$(b) \Rightarrow(a)$. Suppose (a) is false. Since $\langle\mathcal{A}, D\rangle$ is proto-classical, $A$ has at least three members; so suppose we have distinct $a, b \in D$ and $c \notin D$ (the other case is exactly similar). Expand $\mathcal{A}$ to $\mathcal{B}$ by adding three one-place function-symbols, a, $\mathrm{b}$ and + , to its signature, interpreted as follows:

$$
\begin{aligned}
& \mathrm{a} x=a \text { for all } x \in A \\
& \mathrm{~b} x=b \text { for all } x \in A \\
& +x=x \text { for all } x \in A \backslash\{b\}, \text { but }+b=c
\end{aligned}
$$

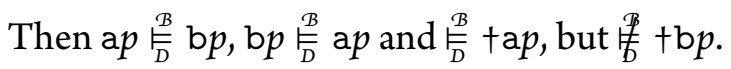

Given our ability to move between algebraic structures and truth-tables, Theorem 6 vindicates my claim that we can pin down the usual truth-tables via the stipulation: It is impossible to add further connectives to our language which would cause violations of the Substitutivity of Equivalents (see $\$ 4$ ). Moreover, Theorem 6 has an immediate corollary: 
the usual connectives are expressively adequate only for the two-valued truth-tables. In many-valued contexts, by contrast, expressive adequacy is a bad thing. ${ }^{6}$

Abstract: Prior's Tonk is a famously horrible connective. It is defined by its inference rules. My aim in this paper is to compare Tonk with some hitherto unnoticed nasty connectives, which are defined in semantic terms. I first use many-valued truth-tables for classical sentential logic to define a nasty connective, Knot. I then argue that we should refuse to add Knot to our language. And I show that this reverses the standard dialectic surrounding Tonk, and yields a novel solution to the problem of many-valued truthtables for classical sentential logic. I close by outlining the technicalities surrounding nasty connectives on many-valued truth-tables.

\section{References}

Carnap, R. (1943). Formalization of Logic. Cambridge MA: Harvard University Press. Church, A. (1944). "Review of Carnap 1943." The Philosophical Review 53.5, pp. 493-8.

- (1953). "Non-normal truth-tables for the propositional calculus." Boletín de la Sociedad Matemática Mexicana 10.1-2.

Fitting, M. (1994). “Kleene's Three Valued Logics and their Children.” Fundamenta Informaticae 20, pp. 113-31.

Koslow, A. (2010). "Carnap's Problem: What is it like to be a normal interpretation of classical logic." Abstracta 6.1, pp. 117-35.

Prior, A. (1960). “The runabout inference-ticket.” Analysis 21.2, pp. 38-9.

Smiley, T. (1996). "Rejection." Analysis 56.1, pp. 1-9.

Williamson, T. (1994). Vagueness. London: Routledge.

${ }^{6}$ Many thanks to Owen Griffiths, Luca Incurvati, Nicholas Jones, Johannes Korbmacher, Arnold Koslow, and two anonymous referees for helpful discussions and comments. 